\title{
Investigation into the role of catabolite control protein $A$ in the metabolic regulation of Streptococcus suis serotype 2 using gene expression profile analysis
}

\author{
XULONG LANG $^{1}$, ZHONGHAI WAN $^{1}$, YING PAN $^{2}$, XIURAN WANG $^{3}$, XIAOXU WANG $^{1}$, \\ ZHAOYANG BU $^{1}$, JING QIAN ${ }^{1}$, HUAZONG ZENG ${ }^{4}$ and XINGLONG WANG ${ }^{1}$ \\ ${ }^{1}$ Key Laboratory of Jilin Province for Zoonosis Prevention and Control, Institute of Military Veterinary, \\ Academy of Military Medical Sciences, Changchun, Jilin 130122; ${ }^{2}$ Changchun Stomatological \\ Hospital, Changchun, Jilin 130042; ${ }^{3}$ School of Life Science, Jilin Agricultural University, Changchun, \\ Jilin 130118; ${ }^{4}$ Shanghai Sensichip Infotech Co. Ltd., Shanghai 200433, P.R. China
}

Received July 21, 2014; Accepted March 20, 2015

DOI: $10.3892 / \mathrm{etm} .2015 .2470$

\begin{abstract}
Catabolite control protein A (CcpA) serves a key function in the catabolism of Streptococcus suis serotype 2 (S. suis 2) by affecting the biological function and metabolic regulatory mechanisms of this bacterium. The aim of the present study was to identify variations in CcpA expression in S. suis 2 using gene expression profile analysis. Using sequencing and functional analysis, CcpA was demonstrated to play a regulatory role in the expression and regulation of virulence genes, carbon metabolism and immunoregulation in S. suis 2. Gene Ontology and Kyto Encyclopedia of Genes and Genomes analyses indicated that CcpA in S. suis 2 is involved in the regulation of multiple metabolic processes. Furthermore, combined analysis of the transcriptome and metabolite data suggested that metabolites varied due to the modulation of gene expression levels under the influence of CcpA regulation. In addition, metabolic network analysis indicated that $\mathrm{CcpA}$ impacted carbon metabolism to a certain extent. Therefore, the present study has provided a more comprehensive analysis of the role of CcpA in the metabolic regulation of S. suis 2, which may facilitate future investigation into this mechanism. Furthermore, the results of the present study provide a foundation for further research into the regulatory function of $\mathrm{CcpA}$ and associated metabolic pathways in S. suis 2 .
\end{abstract}

Correspondence to: Dr Xinglong Wang, Key Laboratory of Jilin Province for Zoonosis Prevention and Control, Institute of Military Veterinary, Academy of Military Medical Sciences, 666 Liuying West Road, Changchun, Jilin 130122, P.R. China

E-mail: wangx1-2006@163.com

Dr Huazong Zeng, Shanghai Sensichip Infotech Co. Ltd., 300 Guoding East Road, Shanghai 200433, P.R. China

E-mail: hzzeng@sensichip.com.cn

Key words: Streptococcus suis serotype 2, catabolite control protein $\mathrm{A}$, gene expression profile, metabolic regulation

\section{Introduction}

Streptococcus suis serotype 2 (S. suis 2) is an important zoonotic pathogen that plays a vital role in the transmission of Streptococcus disease in swine; thus, the bacterium poses a potential threat to susceptible swine populations and humans involved in the raising and processing these animals (1). S. suis 2 is transmitted via open wounds and may be isolated from animal waste and decaying corpses. However, not all types of $S$. suis 2 induce disease, and virulence varies among different strains. S. suis 2 strains may be divided into three types, namely virulent, attenuated and avirulent $(2,3)$. Virulent strains result in evident clinical symptoms, such as acute septicemia, meningitis, arthritis and endocarditis, and may lead to mortality. Virulent S. suis 2 infections in humans are associated with a very high mortality rate $(1,4)$.

Carbon catabolite repression (CCR) is involved in the microbial metabolic process. CCR is a physiological phenomenon in which microorganisms use quick-impact carbon sources, such as glucose among mixed carbon sources, in the process of fermentation. Catabolite control protein A (CcpA) is a key regulator of pleiotropic functions in the process of CCR (5). Furthermore, CcpA is involved in numerous physiological processes, including the regulation of central carbon and nitrogen metabolism, biofilm formation and the expression of virulent genes. CcpA-mediated CCR exists in a number of low-GC Gram-positive bacteria, where it is used to regulate crucial genes in several metabolic pathways through specific functional domains. The CcpA-mediated CCR process exerts a regulatory function by altering $\mathrm{CcpA}$ expression. The specific regulation of CCR may emerge as a notable area of study in the near future (6).

In addition, CcpA is a key regulatory protein of carbon metabolism. Low-GC Gram-positive bacteria adapt to environmental changes by modulating CcpA activity (7). CcpA is a member of the LacI/GalR family of transcriptional regulators. CcpA is not affected by CCR, although the protein regulates multiple metabolic pathways in numerous Gram-positive bacteria (8). Previous studies (9) have identified a number of 
important virulence-associated factors associated with the growth phase in Streptococci, including arcABC, sly, ofs, sao and cps $2 \mathrm{~A}$. The arcABC operon is controlled by CCR, and virulence-associated factors are indirectly regulated by factors in carbon metabolism, such as sucrose and glucose metabolism. In addition, CcpA has been observed to affect virulence through its role as a key regulatory factor of carbon metabolism in a variety of bacteria, including Staphylococcus aureus (10), Streptococcus pneumoniae (11), Clostridium perfringens (12) and S. suis 2 (9).

As a key transcription regulatory factor, CcpA is able to effectively regulate the metabolism of products, such as glucose, in various Gram-positive bacteria. However, the CcpA gene is well-conserved and its CCR function is stable in numerous low-GC Gram-positive bacteria (13). CcpA exhibits similar regulatory functions in the metabolism of Bacillus cereus (14), Staphylococcus xylosus (15), Lactococcus lactis (16), S. pneumoniae (11), Streptococcus mutans (17) and Listeria monocytogenes (18). Therefore, further research into the metabolic mechanisms of bacteria may aid understanding of metabolic processes.

With the increasing research in this field, including S. suis 2 DNA sequences obtained from outbreaks in China (19), the present study investigated the role of $\mathrm{CcpA}$ in functional regulation by analyzing gene expression profiles. In addition, the effect of CcpA on carbon metabolism was investigated using bioinformatics analysis. The current study presents data at a gene expression level to facilitate further study into the role of $\mathrm{CcpA}$ in the metabolic regulation of S. suis 2 and the interaction among various genes.

\section{Materials and methods}

Strains. S. suis 2 and CcpA mutant strains (20) were cultivated to a logarithmic phase in Todd-Hewitt broth medium (Oxoid Ltd., Basingstoke, UK) at $37^{\circ} \mathrm{C}$ in an atmosphere of $5 \% \mathrm{CO}_{2}$. The strains were stored in our laboratory at the Institute of Military Veterinary (Changchun, China) until required for further use.

Extraction and pretreatment. Total S. suis 2 RNA was extracted using a Bacteria total RNA Isolation Kit (Shanghai Sangon Biological Engineering Technology \& Services Co., Ltd., Shanghai, China). Total RNA was examined and quantified using an Agilent 2100 Bioanalyzer (Agilent Technologies, Inc., Santa Clara, CA, USA). The total RNA was used for library construction if the RNA integrity number of $1 \mu \mathrm{g}$ of total RNA was $>7$. Paired-end index libraries were constructed using the NEBNext ${ }^{\circledR}$ Ultra $^{\mathrm{TM}}$ RNA Library Prep kit (Illumina ${ }^{\circledR}$; New England BioLabs, Inc., Ipswich, MA, USA). Large ribosomal RNA among the total DNA was removed using a RiboMinus $^{\mathrm{TM}}$ Transcriptome Isolation kit (Invitrogen Life Technologies, Carlsbad, CA, USA).

cDNA library construction. First and second chains of cDNA were synthesized using ProtoScript II Reverse Transcriptase and Second Strand Synthesis Enzyme Mix (New England BioLabs, Inc.), respectively. Double-chain cDNA was purified using the AxyPrep Mag Polymerase Chain Reaction (PCR) Clean-up kit (Axygen; Corning, Inc., Corning, NY, USA), and End Prep Enzyme Mix (New England BioLabs, Inc.) was used to repair and join the DNA. DNA fragments $(<400 \mathrm{bp})$ with inserts of $\sim 250$ bp were selected using the AxyPrep Mag PCR Clean-up kit, and the PCR products were purified using the AxyPrep Mag PCR Clean-up kit (Axygen; Corning, Inc.). The quality of the library preparations was assessed using the Bioanalyzer 2000 system (Agilent Technologies, Inc.). In addition, the quantity and quality of the PCR products were verified by quantum computing and quantitative PCR (Applied Biosystems Life Technologies, Foster City, CA, USA). Subsequent to confirmation that the library preparations were qualified, sequencing was performed using a HiSeq 2500 Sequencing System (Illumina, Inc., San Diego, CA, USA). The sequencing strategy was SE50 with a length of 1x50 bp (1x50 single-end). Every library consisted of 10 million reads $(0.5 \mathrm{~Gb})$ sequencing products or so with a total length of $>1.0 \mathrm{~Gb}$. Data analyses were performed using HiSeq Control software and GAPipeline-1.6 with a HiSeq instrument (Illumina, Inc.). The Student's t-test was adopted for statistical analyses, where $\mathrm{P}<0.05$ was considered to indicate a statistically significant difference.

Data analyses. After sequencing, raw data of each sample were processed by basecalling andquality analysis.Certainreads were filtered in order to clean the data. Data filtering was performed using the Next Generation Sequencing Quality Control Toolkit version 2.3 (http://59.163.192.90:8080/ngsqctoolkit/). Low-quality and joint sequences were filtered with a QualScore cut-off value of 30 . The $S$. suis 2 sequence was compared with that of S. suis 05AYH33 (GenBank accession no. CP000407.1) using Burrows-Wheeler Aligner version 0.7.5a-r405 (http://sourceforge.net/projects/bio-bwa/files/). Gene quantification was performed using the reads per kilobase per million mapped reads method. Differences in gene expression profiles were analyzed using EdgeR software, version 2.13 (http://bioconductor.org/news/bioc_2_13_release/) with the EdgeR algorithm. More than one difference in gene expression profiles with a false discovery rate of $\leq 0.05$ was considered significant.

Enrichment of Gene Ontology (GO) terms was analyzed using the GO::TermFinder package (http://search.cpan. org/dist/GO-TermFinder/), and a Web Gene Ontology Annotation Plot (WEGO) image was produced at http://wego. genomics.org.cn/cgi-bin/wego/index.pl. Enrichment analysis was performed using the Kyoto Encyclopedia of Genes and Genomes (KEGG) with the KEGG Orthology (KO) Based Annotation System version 2.0 super geometry algorithm (www.genome.jp/kegg/ko.html). All analyses of gene expression profiles and bioinformatics were outsourced to GENEWIZ, Inc. (South Plainfield, NJ, USA).

Metabolic pathway analyses. In order to further elucidate the role of CcpA in the metabolic regulation of S. suis 2, the effects of CcpA on regulation mechanisms were investigated using gene expression profiles based on previous metabonomic analyses (20). In addition, KEGG network data were used to establish a network diagram of interactions among metabolites, genes and proteins. Furthermore, the function of mutants in the interaction of metabolites, genes and proteins in the carbon metabolism pathway were analyzed. 
A

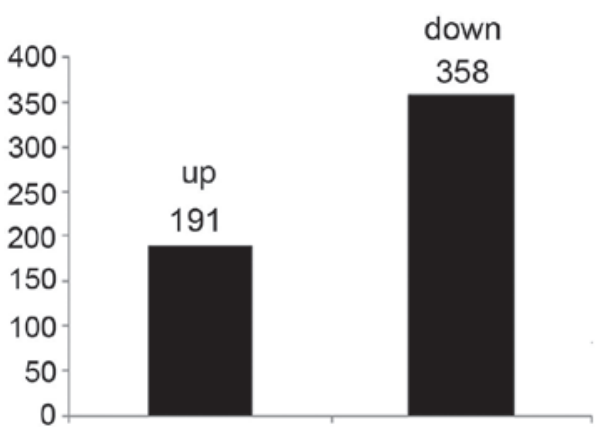

B

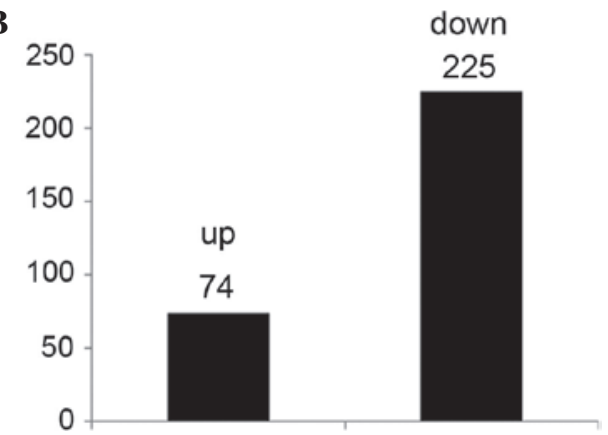

Figure 1. Upregulation and downregulation of (A) differential genes and (B) statistically significant differentially expressed genes.

\section{Results}

Analysis of gene expression profiles. Analysis of gene expression profiles using sequence analysis identification revealed 549 gene differences between S. suis 2 and CcpA mutants (Fig. 1). Differential transcripts selected according to the standard of a significant difference in expression levels identified 299 genes (Fig. 2).

GO information and the functional category of each gene were obtained by GO function analyses of GO using the blast2GO algorithm (http://www.blast2go.com), which facilitated the elucidation of the distribution characteristics of gene function of species or samples at the macro level. Significant enrichment analysis of GO function enriched three GOs $(\mathrm{P} \leq 0.05)$. A total of 1,782 reference genes were annotated in the GO database, while 235 differential genes were annotated. Enrichment results of the differential genes at three levels of $\mathrm{GO}$ were obtained using a hypergeometric algorithm, while enrichment results of the genes with different functions were obtained by WEGO analyses (Fig. 3).

Enrichment analysis of differentially expressed gene pathways. Target protein sequences of $S$. suis 2 were compared with Streptococcus sequences in KEGG GENES using the KEGG Automatic Annotation Server (http://www.genome.jp/kegg/kaas/). Subsequently, the KO number of an identical or similar protein was annotated to the KEGG pathway. Pathway analysis of differential gene expression revealed marked values among the pathways of oxidative phosphorylation, metabolic pathways, one carbon pool by folate, pentose and glucuronate interconversions, and the biosynthesis of secondary metabolites.

Analysis of carbon metabolic regulation. Based on previous studies of associated gene metabonomics, the results demonstrated that CcpA affected carbon metabolism to a certain extent by connecting differential metabolites, genes and proteins (20). According to the network database and the results of the present study, succinic, aspartic and citric acid concentrations decreased and affected the Streptococcus genome under the influence of the CcpA gene, which was verified by previous research from metabolite experiments (20). Furthermore, downregulation of ssu05_1508, ssu05_1065, ssu05_1164, ssu05_1640 and ssu05_2154, and upregulation of ssu05_1885 and ssu05_1839 was observed (Fig. 4). These observations indicated that the carbon metabolism of S. suis 2 was partially regulated by CcpA. Metabolites may alter with modifications in the corresponding genes. Network analysis may facilitate a more comprehensive understanding of the metabolic mechanisms of CcpA in S. suis 2 and provide a foundation for future studies.

\section{Discussion}

Streptococci infections of swine herds are common worldwide and lead to significant financial losses for the swine industry. S. suis 2 is a hazardous zoonotic pathogen that may potentially result in fatal infections in humans. Previously, a massive outbreak of S. suis 2 occurred in Jiangsu and Sichuan provinces, China, resulting in severe swine herd losses $(21,22)$.

CcpA widely participates in the regulation of carbon and nitrogen metabolism in bacteria (17). In addition, CcpA is involved in the specific physiological processes of certain microorganisms, such as the production of spores and solvents, and the expression of virulence genes (23). In S. aureus, CcpA directly regulates and activates the expression of several virulence genes $(10,24)$. Furthermore, in a number of bacteria, deletion of CcpA leads to reduced glycolytic enzyme activity, such as enolase, particularly in infected hosts. Due to specific metabolic associations between particular enzymes and pathogenic virulence, the toxicity of strains without CcpA is notably reduced compared with wild-type parental strains (25). CcpA in Streptococcus pyogenes directly activates the expression of virulence genes $(24,26)$. As a result of the pleiotropic regulatory function of CcpA, numerous metabolic processes are interrupted by knockout of CcpA. Therefore, the association between CcpA structure and function requires further study. Furthermore, mutation analysis may be key to further clarifying the functional domains and active sites of CcpA.

As CcpA is able to regulate genes involved in key metabolic pathways through specific functional domains, the clarification of its regulatory function by transformation of functional domains and complicated pleiotropy into simple specificity requires further investigation. Previous studies investigating S. suis 2 metabonomics indicated that CcpA serves a key function in the regulation of carbon metabolism in amino and nucleic acids and lipids in S. suis 2 (6). Furthermore, pathway analysis indicated that CcpA exerts an indirect influence on the regulation of succinic, nucleic and aspartic acid. In this type of metabolic regulation, the metabolic products are associated with glucose and pyruvic acid, amongst other molecules. As glucose is a readily available carbon source, glucose-induced 


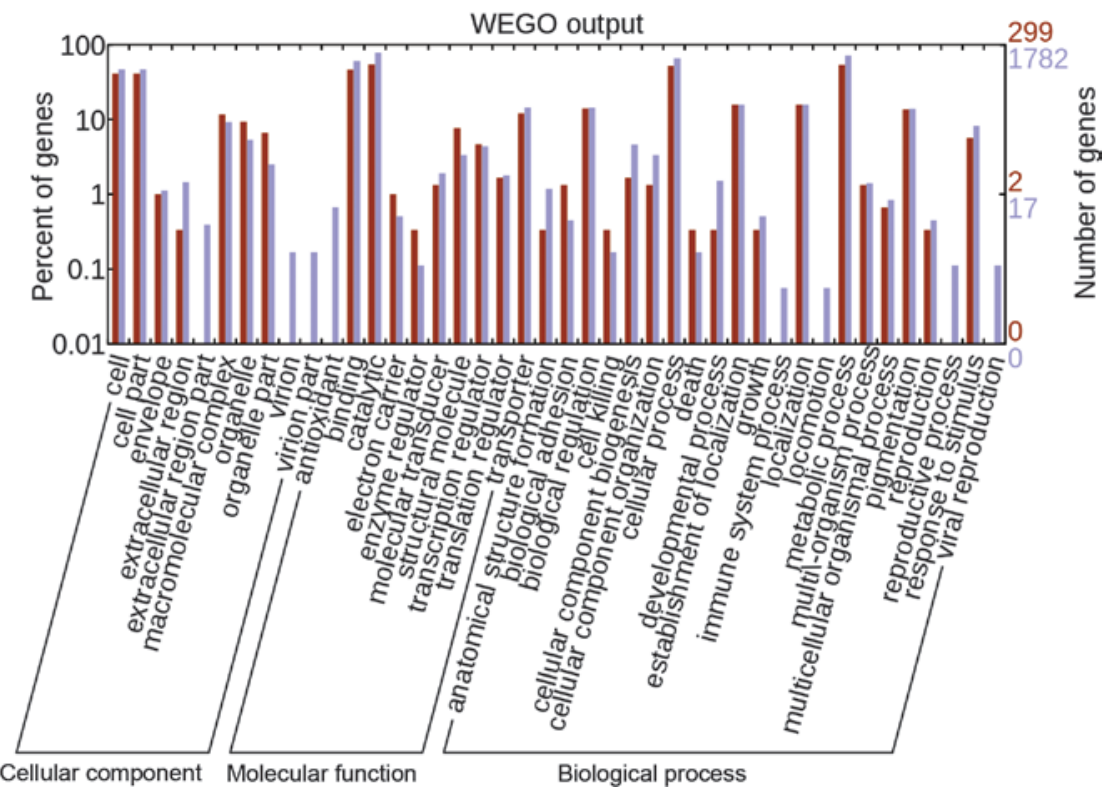

Figure 2. Enrichment analysis of the Gene Ontology function of the differentially expressed gene pathways. WEGO, Web Gene Ontology Annotation Plot.

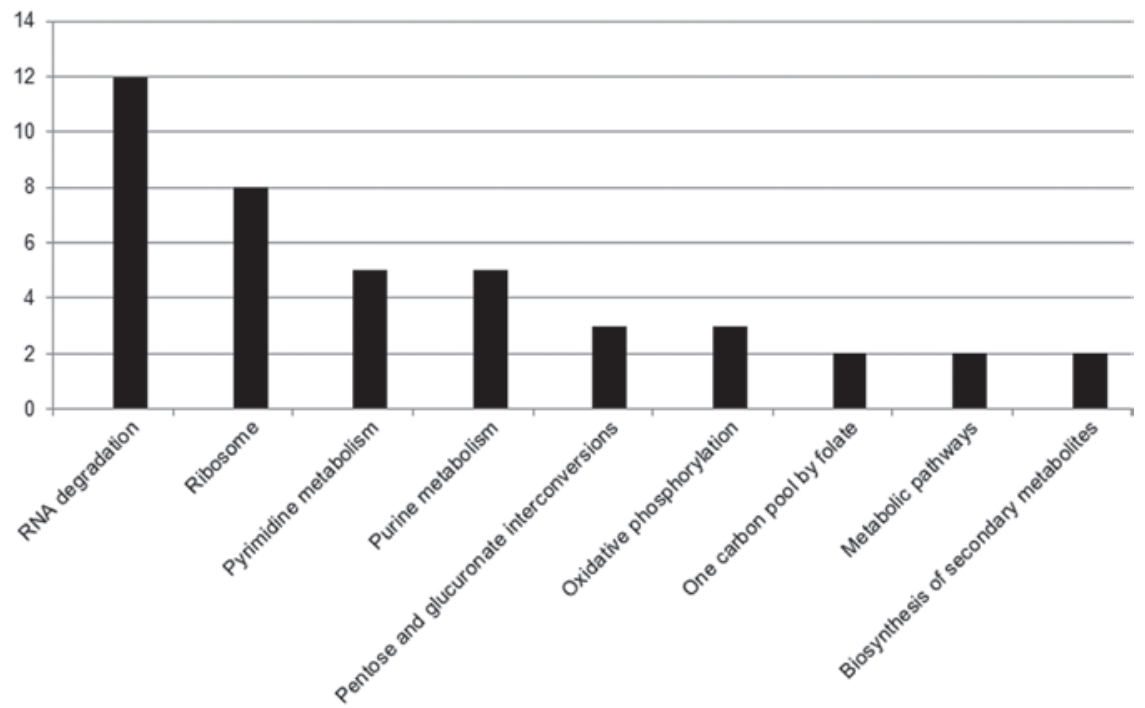

Figure 3. Significant enrichment analysis of the differentially expressed gene pathways.

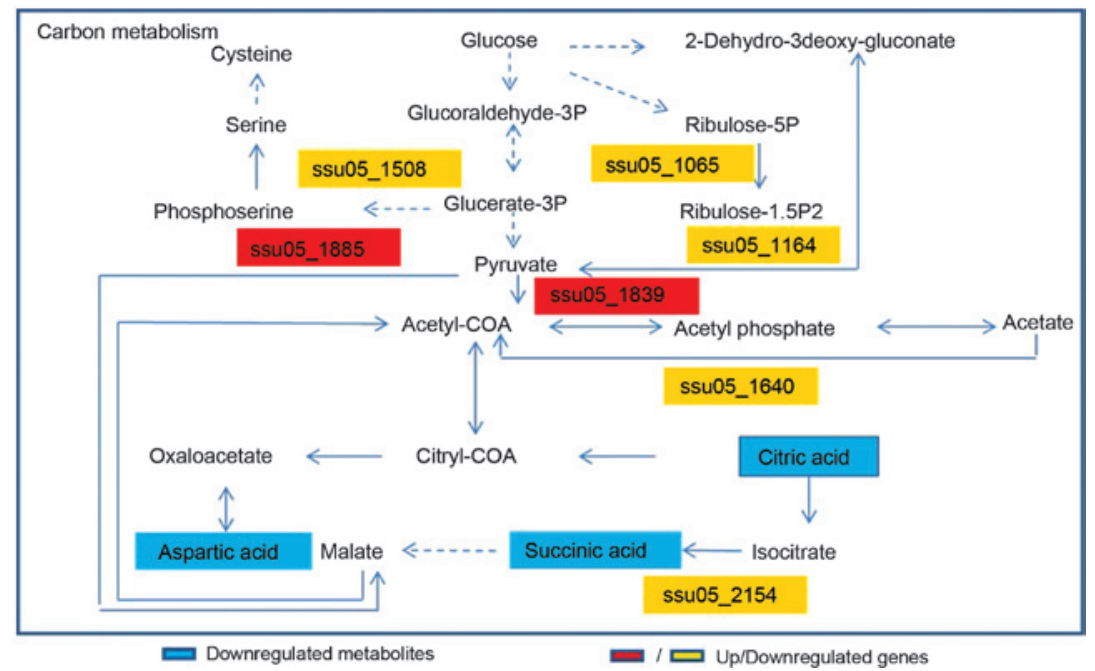

Figure 4. Disrupted metabolic pathways associated with catabolite control protein A in Streptococcus suis serotype 2. 
CcpA-mediated CCR exerts a wide influence on central metabolic pathways $(11,17)$. Thus, CcpA activation and the suppression of certain genes may alter the concentrations of specific metabolites and intermediates. In the present study, reductions in the concentrations of succinic, aspartic and citric acid were also shown to indirectly alter the availability of glucose and similar carbon metabolites, which further affected metabolic regulation and functional changes in S. suis 2. For example, studies on Bacillus subtilis have suggested that CCR relies primarily on the mediation of CcpA (27). In response to environmental glucose, CcpA has been shown to affect catabolite responsive element boxes of target genes via the combined activity of trans-effect factors and cofactors, which subsequently inhibits or activates the transcription of target genes (28). Glucose serves a crucial function in the regulation of numerous central metabolic pathways. The pathway in which CcpA activates or inhibits central metabolism may influence expression levels of particular genes and result in alterations in the secretion of metabolic products. As a signaling molecule, metabolic products indirectly regulate the expression of various genes. Thus, glucose-dependent modifications of central metabolic pathways that alter the distribution of metabolites vary with changing glucose content by altering the transcriptional level of associated genes (29).

In the present study, the Illumina-based analysis of the genome expression of CcpA in S. suis 2 has provided a basis for the further study of the mechanism underlying CcpA activity. In particular, large-scale gene expression analyses offer a suitable analytical method for the study of metabolic and pathogenic mechanisms in bacteria. Gene expression profile analyses were employed to identify genes with varying expression levels involved in metabolic and pathogenic processes of bacteria. Therefore, study into the mechanisms underlying CcpA expression and regulation of possible target genes in S. suis 2 by gene expression profile analysis provides a theoretical basis on which to investigate the role of similar genes in pathogenic mechanisms. Compared with previous studies of CcpA gene transcriptomics in B. subtilis, CcpA was demonstrated to directly or indirectly regulate the expression of several genes and operons, at least to a certain extent $(30,31)$. Similarly, CcpA plays an important role in the regulation of carbon metabolism and virulence regulation in S. suis 2, which has been investigated previously using other methods $(6,32)$. In particular, the study of bacteria in previous studies of functional genomics have been crucial in describing the specific gene expression alterations of a species and have generated large quantities of information concerning the gene expression of cells or tissues under specific conditions. This method is particularly useful in being able to rapidly detect the specific gene organization of a species in a particular state of the expression. However, the results of the present study may facilitate future studies, using the techniques described.

\section{Acknowledgements}

This study was supported by a grant from the National Natural Science Foundation of China (no. 31101790/C1803). Analyses of gene expression profiles and bioinformatics were performed by GENEWIZ, Inc. (Beijing, China). Metabolomic pathway analyses were performed by Shanghai Sensichip Infotech Co. Ltd. (Shanghai, China).

\section{References}

1. Staats JJ, Feder I, Okwumabua O and Chengappa MM: Streptococcus suis: Past and present. Vet Res Commun 21: 381-407, 1997.

2. Busque P, Higgins R, Caya F and Quessy S: Immunization of pigs against Streptococcus suis serotype 2 infection using a live avirulent strain. Can J Vet Res 61: 275-279, 1997.

3. Vecht U, Stockhofe-Zurwieden N, Tetenburg BJ, Wisselink HJ and Smith HE: Virulence of Streptococcus suis type 2 for mice and pigs appeared host-specific. Vet Microbiol 58: 53-60, 1997.

4. Gottschalk M, Xu J, Calzas C and Segura M: Streptococcus suis: A new emerging or an old neglected zoonotic pathogen? Future Microbiol 5: 371-391, 2010.

5. Sanchez S and Demain AL: Metabolic regulation and overproduction of primary metabolites. Microb Biotechnol 1: 283-319, 2008.

6. Willenborg J, de Greeff A, Jarek M, Valentin-Weigand P and Goethe R: The CcpA regulon of Streptococcus suis reveals novel insights into the regulation of the streptococcal central carbon metabolism by binding of CcpA to two distinct binding motifs. Mol Microbiol 92: 61-83, 2014.

7. Kaufman GE and Yother J: CcpA-dependent and -independent control of beta-galactosidase expression in Streptococcus pneumoniae occurs via regulation of an upstream phosphotransferase system-encoding operon. J Bacteriol 189: 5183-5192, 2007.

8. Sumby P, Barbian KD, Gardner DJ, et al: Extracellular deoxyribonuclease made by group A Streptococcus assists pathogenesis by enhancing evasion of the innate immune response. Proc Natl Acad Sci USA 102: 1679-1684, 2005.

9. Willenborg J, Fulde M, de Greeff A, et al: Role of glucose and CcpA in capsule expression and virulence of Streptococcus suis. Microbiology 157: 1823-1833, 2011.

10. Seidl K, Stucki M, Ruegg M, et al: Staphylococcus aureus $\mathrm{CcpA}$ affects virulence determinant production and antibiotic resistance. Antimicrob Agents Chemother 50: 1183-1194, 2006.

11. Iyer R, Baliga NS and Camilli A: Catabolite control protein A (CcpA) contributes to virulence and regulation of sugar metabolism in Streptococcus pneumoniae. J Bacteriol 187: 8340-8349, 2005.

12. Varga J, Stirewalt VL and Melville SB: The CcpA protein is necessary for efficient sporulation and enterotoxin gene (cpe) regulation in Clostridium perfringens. J Bacteriol 186: 5221-5229, 2004.

13. Warner JB and Lolkema JS: CcpA-dependent carbon catabolite repression in bacteria. Microbiol Mol Biol Rev 67: 475-490, 2003.

14. van der Voort M, Kuipers OP, Buist G, de Vos WM and Abee T: Assessment of CcpA-mediated catabolite control of gene expression in Bacillus cereus ATCC 14579. BMC Microbiol 8: 62,2008

15. Jankovic I, Egeter $\mathrm{O}$ and Brückner R: Analysis of catabolite control protein A-dependent repression in Staphylococcus xylosus by a genomic reporter gene system. J Bacteriol 183: 580-586, 2001.

16. Zomer AL, Buist G, Larsen R, Kok J and Kuipers OP: Time-resolved determination of the CcpA regulon of Lactococcus lactis subsp. cremoris MG1363. J Bacteriol 189: 1366-1381, 2007.

17. Abranches J, Nascimento MM, Zeng L, et al: CcpA regulates central metabolism and virulence gene expression in Streptococcus mutans. J Bacteriol 190: 2340-2349, 2008.

18. Behari J and Youngman P: A homolog of CcpA mediates catabolite control in Listeria monocytogenes but not carbon source regulation of virulence genes. J Bacteriol 180: 6316-6324, 1998.

19. Chen C, Tang J, Dong W, et al: A glimpse of streptococcal toxic shock syndrome from comparative genomics of $S$. suis 2 Chinese isolates. PLoS One 2: e315, 2007.

20. Lang X, Wan Z, Bu Z, Wang X, Wang X, Zhu L, Wan J, Sun Y and Wang X: Catabolite control protein $A$ is an important regulator of metabolism in Streptococcus suis type 2. Biomed Rep 2: 709-712, 2014.

21. Tang J, Wang C, Feng Y, et al: Streptococcal toxic shock syndrome caused by Streptococcus suis serotype 2. PLoS Med 3: e151, 2006. 
22. Sriskandan S and Slater JD: Invasive disease and toxic shock due to zoonotic Streptococcus suis: An emerging infection in the East? PLoS Med 3: e187, 2006.

23. Ren C, Gu Y, Wu Y, Zhang W, Yang C, Yang S and Jiang W: Pleiotropic functions of catabolite control protein CcpA in Butanol-producing Clostridium acetobutylicum. BMC Genomics 13: 349, 2012.

24. Poncet S, Milohanic E, Mazé A, et al: Correlations between carbon metabolism and virulence in bacteria. Contrib Microbiol 16: 88-102, 2009.

25. Wang Y, Dang Y, Wang X, et al: Comparative proteomic analyses of Streptococcus suis serotype 2 cell wall-associated proteins. Curr Microbiol 62: 578-588, 2011.

26. Deutscher J, Herro R, Bourand A, Mijakovic I and Poncet S: $\mathrm{P}-\mathrm{Ser}-\mathrm{HPr}$ - A link between carbon metabolism and the virulence of some pathogenic bacteria. Biochim Biophys Acta 1754: $118-125,2005$

27. Seidel G, Diel M, Fuchsbauer N and Hillen W: Quantitative interdependence of coeffectors, CcpA and cre in carbon catabolite regulation of Bacillus subtilis. FEBS J 272: 2566-2577, 2005.
28. Blencke HM, Homuth G, Ludwig H, et al: Transcriptional profiling of gene expression in response to glucose in Bacillus subtilis: Regulation of the central metabolic pathways. Metab Eng 5: 133-149, 2003.

29. Kraus A, Hueck C, Gärtner D and Hillen W: Catabolite repression of the Bacillus subtilis xyl operon involves a cis element functional in the context of an unrelated sequence and glucose exerts additional xylR-dependent repression. J Bacteriol 176: 1738-1745, 1994.

30. Yoshida K, Kobayashi K, Miwa Y, et al: Combined transcriptome and proteome analysis as a powerful approach to study genes under glucose repression in Bacillus subtilis. Nucleic Acids Res 29: 683-692, 2001.

31. Lulko AT, Buist G, Kok J and Kuipers OP: Transcriptome analysis of temporal regulation of carbon metabolism by CcpA in Bacillus subtilis reveals additional target genes. J Mol Microbiol Biotechnol 12: 82-95, 2007.

32. Tang Y, Wu W, Zhang X, Lu Z, Chen J and Fang W: Catabolite control protein A of Streptococcus suis type 2 contributes to sugar metabolism and virulence. J Microbiol 50: 994-1002, 2012. 